\title{
The Galactic Nova Rate
}

\author{
M.DellaValle \\ Dip. di Astronomia,Padova \\ International School of Advanced Studies, Trieste \\ R.Claudi \\ Oss. Astronomico di Padova
}

\begin{abstract}
The recently estabilished values of $M 31$ and $M 33$ nova rate, $r_{M 31}=29 \pm 4$ novae $\mathrm{yr}^{-1}$ (Capaccioli et al.,1989), $r_{M 33}=4 \pm 2$ novae $\mathrm{yr}^{-1}$ (Della Valle, 1988), seems to be at variance with the present estimates for the galactic nova rate, $r=260$ novae $\mathrm{yr}^{-1}$ (Sharov,1972) and $r=73 \pm 24$ novae $\mathrm{yr}^{-1}$ (Liller and Meyer,1987), if we take into account the luminosity class and the Hubble type of the Galaxy. Assuming the nova production per unit of B-luminosity about constant for spiral galaxies and adopting the derived values of the nova rate of M31, M33 and LMC as calibrators, we estimate a galactic nova rate of $r_{G a l}=15 \pm 5$ novae $\mathrm{yr}^{-1}$.
\end{abstract}

The novae rate in extragalactic systems is closely related to the following topics:

a) the determination of the space density of the classical novae;

b) the estimate of the recurrence periods between two following outbursts;

c) the contribute to the interstellar medium by the ejecta (van den Bergh, 1988);

d) possible dependence of the nova rate on the Hubble type of the parent galaxies;

e) birth place of the novae: bulges of the galaxies and/or globular clusters (Ciardullo et al. 1987).

For the same reasons it appears quite attractive to give a correct estimate of the galactic nova rate. However the only recent available estimate of the galactic novae rate is based on the poorly reliable determination obtained by Liller and Meyer (1987) of $R_{G a l}=73 \pm 24$ novae/yr. This value is derived from an observed rate of $r \simeq 3.7$ novae (including 2 recurrent novae, $3 \mathrm{DN}$ and 1 Nova Like) and by applying a global corrective factor of $\simeq 20$ in order to take into account seasonal, fade-out, weather, geometric and overlooking effects. This figure appears conflicting with the quite surprising Sharov's determination (1972) of $R_{G a l} \simeq 260$ novae/yr, too.

We shall now try to determine the galactic nova rate interpolating between the rates observed in M31 ( $R=29 \pm 4$ novae/yr, Capaccioli et al., 1989), M33 ( $R=4 \pm 2$ novae/yr, Della Valle, 1988) and LMC ( $R=2 \pm 1$ novae/yr Graham 1979) (Fig. 1). This assumption is supported by the observations (see Table 1, col 7) and moreover it seems reasonable to apply to the Galaxy because of its intermediate Hubble type among the ones exhibited by the calibrators. In Table 1 we report the data utilized in order to draw the $B$ luminosity of the galaxies and the nova rate per unity of $B$ luminosity $\left(10^{10} L_{\odot}\right)$. Assuming a $L_{B} \simeq 2 \times 10^{10} L_{B_{\odot}}$ for the Galaxy (van den Bergh 1988), the available data suggest a galactic nova rate of $R=15 \pm 5$ novae/yr. 
Table 1

\begin{tabular}{lrrrrrr}
\hline \hline Galaxy & $B_{T}$ & $A_{\urcorner}^{0}$ & $A^{i}$ & (m-M) $)^{-}$Novac/yr & \multicolumn{1}{c}{$\begin{array}{c}\text { Novac/yr } \\
10^{10} L_{B_{\odot}}\end{array}$} \\
\hline M31 & 4.38 & 0.32 & 1.03 & $24.30 \pm 0.20$ & $29 \pm 4$ & $5.8 \pm 0.8$ \\
M31 bulgo $_{\text {B }}$ & 5.22 & 0.32 & 1.03 & $24.30 \pm 0.20$ & $24 \pm 4$ & $8.9 \pm 0.8$ \\
M33 & 6.26 & 0.18 & 0.45 & $24.60 \pm 0.30$ & $4 \pm 2$ & $7 \pm 3$ \\
LMC & 0.63 & 0.26 & 0.16 & $18.65 \pm 0.20$ & $2 \pm 1$ & $6 \pm 3$ \\
Virgo & 9.52 & & & $31.30 \pm 0.40$ & $180 \pm 60$ & $24 \pm 8$ \\
Galaxy & & & & & $73 \pm 24$ & $36 \pm 10$ \\
\hline
\end{tabular}
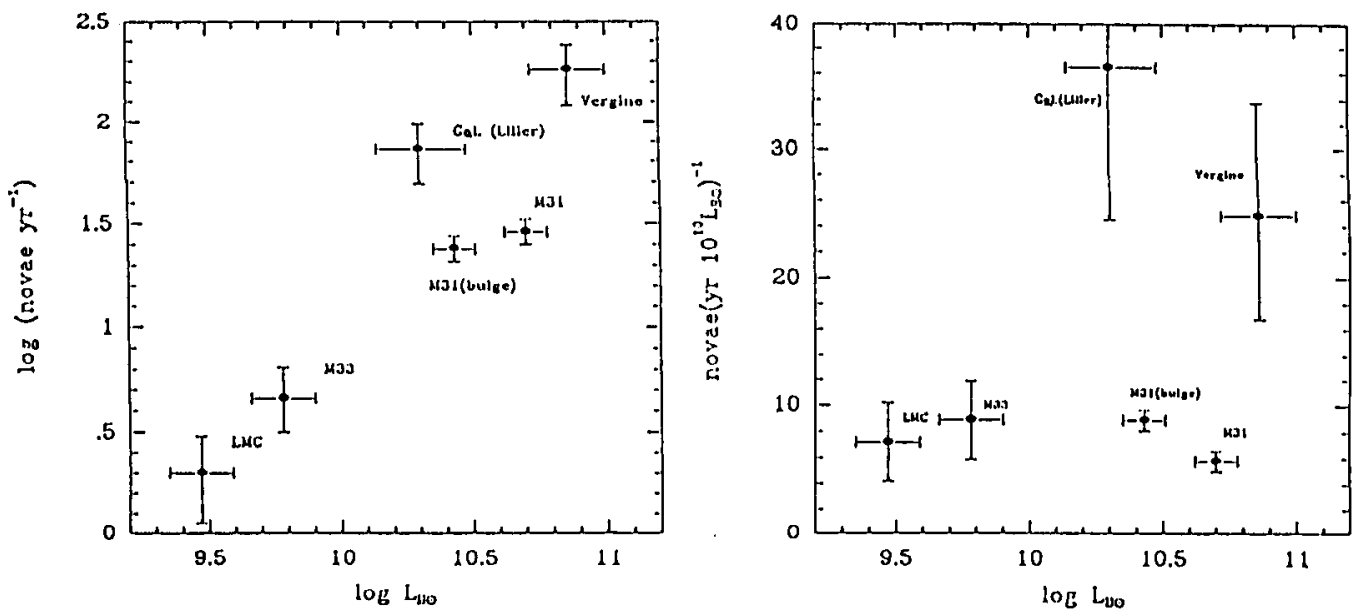

fig. 1

\section{References}

- Capaccioli,M., Della Valle M., D' Onofrio M., Rosino L. 1989a, A.J. 07,622

- Ciardullo R., Neill J. Jacoby G., Shafter A. 1987, Ap. J. 318,520

- Della Vallc M., 1988, proc.of the Symp. The Extragalactic Distance Scale, eds. S. van den Bergh and C. Pritchet

- Graham J.a., 1979 in Changing Trends in Variable Stars Rescarh IAU Colloquium 46, eds F.M. Bateson, J. Smak, I.H. Urch, pag. 96

- Liller W., Mayer B., 1987, Pub.a.s.p.,00,606

- Sharov A.S., 1972, Soviet Astron.,16,41

- van den Bergh S.,1988, Pub.A.S.P., 100,8 